\title{
Gobiernos y ciudadanías abiertas en la era digital: análisis socio-técnico sobre la participación política y la gestión de bienes intelectuales ${ }^{1}$
}

Open government and citizenship in the digital age: sociotechnical analysis on political participation and management of intellectual property

Ariel Vercelli ${ }^{2}$

Artigo recebido em para publicação em out/2012 e aceito para publicação em nov/2012.

\section{Resumen:}

En este artículo se analiza el concepto de 'gobierno abierto' ['open government'] y su relación con la gestión estratégica de derechos ciudadanos en la era digital. Se caracterizan la gestión de datos, informaciones públicas y obras intelectuales y se presentan casos a nivel global [EE.UU., Reino Unido, Australia, Holanda, España y Argentina]. El artículo busca fortalecer el ejercicio ciudadano de los derechos de libre acceso y disponibilidad sobre datos, informaciones públicas y obras intelectuales

en

América

Latina.

Palabras clave: ciudadanías y gobiernos abiertos. Tecnologías digitales. Gestión de derechos. Datos. Informaciones públicas. Obras intelectuales.

\section{Abstract:}

This article discuss the concept of 'open government' and its relationship to the strategic management of citizen rights in the digital age. It characterizes the management of data, public information and intellectual works, by presenting some global cases [USA, United Kingdom, Australia, Netherlands, Spain and Argentina]. This article seeks to strengthen the citizen exercise of the free access rights and availability of data, public information and intellectual works in Latinamerica.

Keywords: citizenships and open government. Digital technologies. Rights Management. Data. Public Information. Intellectual works.

\footnotetext{
${ }^{1}$ Artigo originalmente apresentado na IX Jornadas Latinoamericanas de Estudios Sociales de la Ciencia y la Tecnología, México, 2012.

${ }^{2}$ Ariel Vercelli es Investigador Asistente del 'Consejo Nacional de Investigaciones Científicas y Técnicas' (CONICET) y Presidente de 'BIENES COMUNES Asociación Civil'. Es Doctor em Ciencias Sociales y Humanas de la 'Universidad Nacional de Quilmes' (UNQ), Magíster en Ciencia Política y Sociología, Escribano y Abogado. Email: arielvercelli@arielvercelli.org.
} 


\section{Introducción}

En el artículo se analiza -desde un enfoque socio-técnico- el concepto de 'gobierno abierto' [del inglés 'open government'] y su relación con el ejercicio y gestión de derechos ciudadanos. Si hace varios años que los gobiernos comienzan a autoproclamarse "abiertos", entonces, ¿cuáles son los cambios jurídico-políticos y tecnológicos que la era digital trae para los ciudadanos? ¿Los gobiernos abiertos se corresponden con ciudadanías abiertas? En el artículo se analiza como aquello que es "abierto" mantiene una relación profunda con las tradiciones 'abiertas / libres' provenientes de la cultura de Internet. El artículo es aún exploratorio y forma parte de una investigación más amplia donde se analiza empíricamente las relaciones que se producen entre las tecnologías digitales y las regulaciones / formas jurídico-políticas. El artículo busca mejorar y fortalecer la participación política ciudadana, la democracia, la distribución de la riqueza intelectual y el ejercicio de los derechos ciudadanos de libre acceso y disponibilidad sobre la producción de carácter público.

\section{El renacimiento de los gobiernos electrónicos "abiertos"}

El desarrollo de las tecnologías digitales, la expansión de Internet y el crecimiento de las redes de telefonía móvil han favorecido profundos cambios en las formas políticas a nivel global ${ }^{3}$. Estas tecnologías y las formas de gobierno ya tienen una larga historia. Las promesas, búsquedas y reivindicaciones de mejores formas de gobierno estuvieron presentes desde los inicios de la digitalización: desde la informatización de las administraciones públicas hasta los más sofisticados y ambiciosos proyectos de gobierno electrónico ${ }^{4}$ (Berners Lee, 2000; Castells, 2001; 2009; Naser y Concha, 2012). En la actualidad, tal vez con más exigencias, urgencias y escepticismos, todavía sigue en pie la pretensión de construir gobiernos más democráticos, republicanos, una ciudadanía digital fuerte y, en suma, toda una nueva y mejor relación entre los ciudadanos y sus gobernantes.

\footnotetext{
3 En ningún caso estas tecnologías han sido neutras. Por un lado, han renovado y fortalecido [y pueden fortalecer y enriqucer aún más] el ejercicio directo e inmediato de derechos ciudadanos fundamentales: la libertad de expresión, el derecho a peticionar a las autoridades, el derecho de autor y de copia, el derecho de libre acceso y disponibilidad de la información pública (Lessig, 2001, Benkler, 2006; Vercelli, 2009). Por el otro, lejos de una neutralidad tecnológica, estas mismas tecnologías también han servido [y pueden servir] para generar nuevas formas de censura, menoscabos a los derechos intelectuales, la desaparición de la privacidad, formas autoritarias de control o la privatización de datos e informaciones de carácter público (Boyle, 2008; Lessig, 2008; Vercelli, 2009), Ciertas configuraciones sociotécnicas pueden corromper profundamente las actuales formas de gobierno y contribuir al desarrollo de gobiernos despóticos y totalitarios.
}

${ }^{4}$ Además de gobierno electrónico, con diferencias y matices, también se han utilizado los conceptos de telegobierno, e-gobierno, administración electrónica, gobierno en línea o gobierno digital. 
La idea de un 'gobierno (electrónico) abierto' ha surgido recientemente como una vía, una alternativa, como un camino posible que conduzca a la construcción de mejores formas de gobierno en la era digital. Estas nuevas formas de gobierno procuran ser más transparentes, confiables, participativas y colaborativas en la administración de la cosa pública (Obama, 2009; Orszag, 2009; Irekia, 2010a; Naser y Concha, 2012). Se presentan a nivel internacional como un nuevo intento de llevar mayor apertura, diálogo y participación ciudadana a la política. En este sentido, los gobiernos abiertos parecen enfrentarse al secretismo, la clausura y, sobre todo, a la brecha que separa los ciudadanos y sus gobernantes dentro del sistema de respresentación política (Orszag, 2009; Irekia, 2010a, Ramírez Alujas, 2010).

En pocos años los gobiernos abiertos se pusieron "de moda". El primero de los gobiernos (autodenominado) "abierto" a nivel mundial se inició a principios de 2009 en los Estados Unidos de Norteamérica (EE.UU.). El actual Presidente de EE.UU., Barack Obama, ha sido el principal impulsor de esta modalidad de gobierno electrónico. Obama inició su proyecto de gobierno abierto desde la misma campaña hacia la presidencia. Para ello, utilizó blogs y redes sociales (como Facebook y Twitter), diseñó toda una nueva forma de financiar su campaña política, convocó insistentemente a la participación ciudadana y, en pocos meses de su gestión, resignificó profundamente las formas de gestionar la cosa pública. Para Obama, la apertura de su gestión democratizó los datos e informaciones públicas y mejoró la vida cotidiana de los ciudadanos (Obama, 2009; Orszag, 2009).

Los gobiernos abiertos se caracterizan inicialmente por, al menos, utilizar las tecnologías digitales y las plataformas tecnológicas para buscar mayores y mejores niveles de: [a] transparencia en las formas de gestionar el patrimonio público; [b] participación activa de los ciudadanos en la elaboración y deliberación de leyes y políticas (en forma directa o a través de organizaciones de la sociedad civil); [c] colaboración entre gobiernos, ciudadanos, partidos políticos, sindicatos, empresas, organizaciones de la sociedad civil, etc. Estos tres elementos están presentes en todas las iniciativas a nivel global, son los pilares sobre los que se asienta la apertura en las formas de gobierno (Orszag, 2009; Irekia, 2010a, Vercelli, 2010).

¿Existen otros elementos comunes que permitan caracterizar los gobiernos abiertos? Un cuarto elemento presente en estas iniciativas es la [d] gestión de bienes intelectuales. Los bienes intelectuales, estas formas de valor, se expresan de diferentes maneras y pueden presentarse a través de los más diversos soportes [materiales]. En este sentido, las experiencias de gobiernos abiertos implican siempre una gestión estratégica de las diferentes formas de valor intelectual. Esta gestión se divide, al menos, en tres formas de valor bien definidas: [d1] gestión de datos (bases de datos), [d2] gestión de informaciones públicas y [d3] de obras intelectuales (por ejemplo, software, música, artículos de investigación científica o informes técnicos de la administración pública). Muchas de estas formas típicas de 
expresión y gestión de bienes intelectuales se corresponden con regulaciones específicas.

El concepto de gobierno abierto aún no tiene un desarrollo sistemático ni es una doctrina política. Es un concepto en construcción, ambiguo, polisémico, e, incluso, redundante. Está atravesado por infinitas preguntas y, por el momento, las experiencias empíricas a nivel mundial generan hoy más dudas que certezas. Entre ellas, un primer nivel de preguntas cuestiona: ¿qué significa realmente que un gobierno sea abierto? Puede parecer sorprendente e insólito, pero no queda claro a nivel internacional qué significa esta apertura en las formas políticas. En igual sentido, ¿por qué gobiernos que ya son democráticos y republicanos deben pasar a ser también abiertos? ¿Cómo se relacionan operativamente "lo abierto" con la transparencia, la participación, la colaboración y la gestión de los bienes intelectuales (datos, informaciones públicas y obras intelectuales?

Por estos días se habla mucho gobiernos abiertos, aunque, en proporción, muy poco se habla de su correlato político: las ciudadanías abiertas o las nuevas formas de participacion política ciudadana en la era digital. Es decir, un segundo nivel de preguntas, intimamente relacionadas a las primeras, cuestionan qué tipo de relación se presenta entre las tecnologías digitales y estas nuevas formas abiertas de gobierno. En concreto, ¿estas formas abiertas de gobierno están generando también ciudadanías abiertas? ¿El renacimiento de los gobiernos electrónicos abiertos está generando también un correlato jurídico-político y tecnológico sobre la gestión de derechos ciudadanos? ¿Cuánto ha cambiado la capacidad que tienen las ciudadanas y los ciudadanos para participar políticamente a través de Internet o la telefonía móvil? ¿Dónde -a través de qué canales- se produce la participacion política ciudadana en los gobiernos abiertos? ¿Se pueden medir los niveles de participación y colaboración?

Un tercer nivel de preguntas cuestiona qué tipo de gestión digital de derechos intelectuales pueden hacer los ciudadanos en los esquemas de gobiernos abiertos. En concreto, ¿cuál es el carácter que tienen los datos, las informaciones públicas y las obras intelectuales que se producen en cumplimiento de las funciones públicas? ¿Son de carácter privativo, público o común? ¿Qué niveles de acceso y disponibilidad tienen los ciudadanos, las comunidades, las empresas u otros Estados? ¿Los datos se abren a los ciudadanos o sólo se preparan para que empresas y grandes corporaciones puedan ofrecer sus servicios? ¿Estos modelo de gestión de bienes intelectuales fueron creados y diseñados para sostener los gobiernos abiertos 0 , en realidad, provienen de la misma estructura y tradición de Internet?

\section{La cultura 'abierta / libre' de Internet llega a los gobiernos}

Las características descritas de los gobiernos abiertos están íntimamente relacionadas con la arquitectura, la política y la cultura que ha definido el desarrollo de las tecnologías digitales e Internet a lo largo de su historia. El 
concepto de gobierno abierto también deriva de la apertura de las redes electrónicas y sus protocolos, de la libertad / apertura de los movimientos por el software libre (del inglés 'free software') y software de 'fuente abierta' (del inglés 'open source'), del movimiento por el acceso abierto (del inglés 'open access'), de los movimientos por la liberación y disponibilidad de la cultura (del inglés 'free culture') y, en suma, de toda una cultura abierta / libre que caracterizó el desarrollo de Internet desde sus inicios (Castells, 2000, 2009; Stallman 2004, Lessig, 2006; Vercelli, 2004, 2009; Boyle, 2009). En la era digital, aquello que es abierto / libre también construye formas de gobierno.

El desarrollo de las tecnologías digitales, la expansión de Internet y las arquitecturas abiertas, libres y de producción colaborativa entre pares han permitido que, como nunca antes en la historia de la humanidad, millones y millones de personas (usuarios-finales, ciudadanos) a nivel global puedan informarse, expresarse, gestionar sus derechos ciudadanos y colaborar entre pares para producir, disponer y compartir todo tipo de bienes $^{5}$ intelectuales ${ }^{6}$. Por ello, una de las hipótesis que inicialmente guía esta investigación es que todo aquello que es "abierto" dentro de estas nuevas formas políticas mantiene una relación profunda e íntima con las tradiciones 'abiertas / libres' sobre las que se desarrollaron las tecnologías digitales y que permitieron la expansión de las redes electrónicas distribuidas como Internet. A su vez, subsidiariamente, las nuevas formas políticas abiertas en la era digital mantienen una relación (estrecha, íntima, de retroalimentación) de co-construcción con las tecnologías digitales disponibles para el ejercicio de los derechos ciudadanos y la gestión de gobierno.

Lejos de ser pacíficos, los cambios en las formas de gobierno y sus correlatos en las formas de gestión de derechos ciudadanos estuvieron (y aún están) atravesados por todo tipo de tensiones, negociaciones, luchas e imposiciones por parte de los diferentes grupos sociales. Muchas de estas tensiones y negociaciones son cotidianas, se encuentran presentes a cada clic de mouse, al momento de escribir un correo electrónico, una entrada en un blog, dejar un comentario en una red social, navegar una página web, chatear, intercambiar archivos en redes de pares, construir un avatar en un mundo virtual, enviar un mensaje de texto por teléfonos móviles, jugar en

\footnotetext{
${ }^{5}$ El concepto de 'bien / bienes' indica aquello que tiene [o puede tener] un valor, un interés, una utilidad, un mérito y que, a su vez, recibe [o puede recibir] protección jurídica. Así, los bienes son todas aquellas 'cosas materiales' o 'entidades intelectuales' en cuanto objetos de derecho. La definición de 'bien / bienes' alcanza a todo lo que sea o pueda ser jurídicamente tutelado más allá del reconocimiento expreso en una ley positiva o de las interpretaciones jurídicopolíticas dominantes (Vercelli, 2009).

${ }^{6}$ A diferencia de los bienes de calidad material [o cosas], los datos, las informaciones o las obras intelectuales se definen como bienes de calidad intelectual. Si bien se expresan en soportes materiales, estos bienes intelectuales [más aún los que se encuentran en formatos digitales] son abundantes y pueden compartirse libremente entre diferentes grupos sociales sin generar relaciones de escasez. Por su calidad los bienes intelectuales tienen todos un carácter común. Se puede disponer de ellos para cualquier propósito de forma directa, inmediata y sin mediaciones. (Vercelli, 2009).
} 
red a través de consolas de videojuegos o participar políticamente a través de un portal gubernamental. A pensar de su cotidianeidad y distribución, la ciudadanía no es plenamente conciente de las mediaciones tecnológicas, es decir, de cuánto se ven afectados sus derechos ciudadanos por el uso de ciertas las tecnologías y plataformas digitales.

\section{El enfoque socio-técnico sobre la gestión de derechos ciudadanos}

El artículo y esta línea de investigación se basan en un análisis sociotécnico (Bijker, 1995; Thomas, 2008; Vercelli, 2009). Para el enfoque sociotécnico el significado de un artefacto tecnológico (en sus múltiples y diversas expresiones) no puede encontrarse dentro del mismo artefacto. Un artefacto tecnológico no es auto-explicativo, no tiene razones internas, inmanentes o intrínsecas que lo expliquen por sí mismo más allá de sus diversas inter-relaciones sociales, técnicas, económicas, políticas que lo constituyen históricamente. Este enfoque socio-técnico reconstruye analíticamente tanto el momento dónde los artefactos tecnológicos son partes constitutivas de las relaciones sociales, políticas, económicas como a su vez- el momento en que estas diversas relaciones se expresan, codifican y materializan en artefactos tecnológicos. Es decir, analiza cómo las tecnologías son construidas socialmente y cómo las sociedades son construidas tecnológicamente.

El análisis socio-técnico permite [des]construir la interrelación entre lo social y lo tecnológico. Este enfoque busca evitar [y superar] las posiciones deterministas y lineales tanto socio-jurídico-políticas como tecnológicas. El marco de análisis socio-técnico propuesto se ubica dentro de un abordaje constructivista y relativista del desarrollo tecnológico (Thomas, 1999). El abordaje es constructivista puesto que busca captar el momento en que, a través de sus acciones, los diferentes grupos sociales (más o menos relevantes) van construyendo problemas y soluciones en función de la protección o garantización de sus intereses. A su vez, el abordaje es relativista puesto que dentro de este enfoque no existen tecnologías, regulaciones, políticas, bienes 0 cualquier tipo de construcciones sociales, que se mantengan sin cambios, que sirvan en todo tiempo y lugar o puedan considerarse universales. Estas construcciones son socio-históricamente situadas (Thomas, 2008, Vercelli, 2009).

El abordaje socio-técnico se utiliza para analizar cómo se construyen las experiencias de gobierno abierto y cómo se relacionan los derechos ciudadanos y las tecnologías digitales que se utilizan para su gestion. A través de estas relaciones se busca analizar cómo y de qué forma cada una de las relaciones jurídicas, bienes, artefactos, tecnologías o portales de gobierno abierto que pueden analizarse son la resultante de un proceso continuo de luchas, discusiones, negociaciones y re-definiciones por parte de los diferentes grupos sociales relevantes (Thomas, 2008; Vercelli, 2009). Los 'grupos sociales relevantes' son aquellos grupos de actores que atribuyen significados (Bijker, 1995: 46): entre otros, 
ciudadanos que usan redes sociales, gobiernos (nacionales, provinciales y municipales) que implementan soluciones de gobierno abierto, empresas de desarrollo de software, corporaciones transnacionales, académicos dedicados al tema, ingenieros, los partidos políticos, ONGs) ${ }^{7}$.

A través de este enfoque se busca captar el conjunto de relaciones que hacen que se diseñen tecnologías para gestionar datos, que se sancionen leyes sobre acceso a las informaciones públicas, que se produzcan licencias para liberar obras intelectuales o que se construyan plataformas orientadas a la participación política ciudadana. Por ello, dentro de esta investigación, el funcionamiento o no-funcionamiento de una solución de gobierno abierto no responde a una propiedad intrínseca. Por el contrario, es el resultado de un complejo proceso de construcción sociotécnica en el que se evidencian las negociaciones e imposiciones de significados atribuidos por los diferentes grupos sociales ${ }^{8}$. El funcionamiento o no-funcionamiento de una solución de gobierno abierto o de una plataforma comunitaria de gestión de derechos ciudadanos es el resultado de un proceso complejo en el que se evidencia su éxito o fracaso, su viabilidad o abandono, según las negociaciones e imposiciones de significados atribuidos por los diferentes grupos sociales ${ }^{9}$.

Las relaciones que se producen entre las regulaciones ${ }^{10}$ y las tecnologías son profundas. En ambas instancias se codifican intereses,

\footnotetext{
${ }^{7}$ El análisis socio-técnico permite enfocar (seguir) los grupos sociales que, con su accionar, van construyendo tanto las tecnologías como las regulaciones. En este sentido, se puede observar como las regulaciones o las tecnologías son construidas por las mismas personas (ciudadanos) que son usuarios-finales de una red distribuida de telefonía móvil, lectores de una biblioteca de una agencia gubernamental, usuarios hogareños de la televisión digital terrestre o ciudadanos que utilizan servicios de gobierno abierto. En esta investigación se pretende mostrar cómo es que las regulaciones y las tecnologías son construidas por la acción de los diferentes grupos sociales y hacen que éstas tengan una composición heterogénea, que sean una construcción híbrida (Bijker, 1995; Callon, 1998, Latour, 2004).
}

${ }^{8}$ El análisis del proceso de imposición y negociación de estos significados permite comprender el desarrollo histórico, el cambio, o bien, el éxito o fracaso de cualquiera de estas construcciones (Thomas, 2008). Según Bijker (1995), la existencia de diversos significados atribuidos a un artefacto por parte de los distintos GSR es un indicio su 'flexibilidad interpretativa' en un tiempo y lugar determinados. Esta flexibilidad interpretativa aumenta o disminuye a medida que se negocian, discuten, consensúan o imponen diferentes significados sobre un artefacto (Bijker, 1995).

${ }^{9}$ La noción de funcionamiento puede definirse como un emergente, como algo contingente, relativo a un tiempo-espacio determinado y sujeto a una diversidad de fuerzas y tensiones [políticas, técnicas, sociales, económicas o legales]. En esta investigación el concepto de adecuación socio-técnica permite describir por qué una política de gobierno abierto, unas regulaciones o determinadas tecnologías "funcionan". El concepto de adecuación es complementario de concepto de funcionamiento. Por lo general, las tecnologías "funcionan" o "no-funcionan" por un compromiso entre la asignación y negociación de sentido de los grupos sociales y la materialidad de los artefactos (Thomas, 2008).

\footnotetext{
${ }^{10} \mathrm{Al}$ igual que ocurre con las tecnologías, para esta investigación las regulaciones también tienen una composición heterogénea. Están compuestas por leyes, modelos de gestión, normas sociales de los usuarios, costumbres, condiciones de mercado, políticas, arquitecturas, tecnologías y, en suma, todo lo que haga funcionar las construcciones jurídicas. A diferencia de
} 
valores y posiciones políticas de los grupos sociales (Vercelli, 2004, 2009). Por ello, en este artículo se propone analizar las experiencias de gobiernos abiertos enfocando aquello que ocurre con la gestión digital de los derechos ciudadanos. Los procesos políticos de codificación y de-codificación pueden verse sintetizados en las formas de gestión, plataformas web o redes sociales que son, simultáneamente, a un mismo tiempo, tanto tecnologías como regulaciones. En este sentido, tanto las regulaciones como las tecnologías se orientan hacia el control de procesos, conductas humanas o espacios (Vercelli, 2004). Ambas buscan ajustar, balancear, estabilizar el relacionamiento social. Son parte de un proceso complejo de intervención política sobre las conductas y los espacios a nivel social.

Específicamente, las regulaciones y las tecnologías digitales mantienen una relación estrecha, íntima, profunda, frente a las diferentes formas de control político y social. Son parte de un proceso mayor donde ambas se articulan, cambian simultáneamente, co-evolucionan, co-varían a través del tiempo". Esta relación se define como un proceso de "coconstrucción" entre regulaciones y tecnologías ${ }^{12}$. Así, el concepto de coconstrucción permite analizar cómo, a través del mismo proceso [en el mismo momento, en el mismo acto, simultáneamente], se construyen regulaciones para tecnologizar la sociedad y se construyen tecnologías para regular y gobernar las sociedades.

La estrategia teórico-metodológica de esta investigación consiste así en analizar los casos de gobierno abierto desde un enfoque sociotécnico, es decir, enfocando los procesos de co-construcción que se producen entre regulaciones de derechos intelectuales y las tecnologías

\footnotetext{
lo que indica el sentido común, una regulación no es sólo una ley o un conjunto de leyes. A diferencia de las leyes y otras expresiones jurídicas, las regulaciones siempre tienen una faz práctica que debe ser contrastada empíricamente. Más que una proyección o la expresión de intenciones, son el resultado de un proceso complejo que va delineando las formas repetidas, las formas medias del acontecer social. Una regulación, una política o cualquier artefacto tecnológico funcionan o no-funcionan a partir de las negociaciones de sentidos, de los usos y de las prácticas de los diferentes grupos sociales.
}

${ }^{11}$ En los entornos digitales, las regulaciones y las tecnologías mantienen históricamente una relación estrecha, complementaria y de retro-alimentación (Kapor, 1990; Barlow, 1994; 1996; Mitchell, 1995). En el libro 'Código y otras leyes del Ciberespacio', Lawrence Lessig (2006) fue uno de los primeros en explicitar cómo los "códigos eran la ley" ["code is law"] dentro de los entornos digitales y en mostrar cómo este proceso afectaba políticamente la red de redes. Los estudios sobre las regulaciones en Internet comenzaron a extender su análisis más allá de la esfera estrictamente legal. Internet comenzó a observarse como una red regulada a través todo tipo de leyes, arquitecturas, costumbres, reglas de mercado y tecnologías (Lessig, 2006; Vercelli, 2004; Benkler, 2006). El diseño de las tecnologías comenzó a ser uno de los puntos de análisis de las regulaciones (Feenberg, 1999; 2002) y emergió, con mayor claridad, la íntima relación entre la producción de regulaciones de derechos intelectuales y las tecnologías digitales diseñadas para gestionar estos derechos (Vercelli y Thomas, 2007; Vercelli, 2009).

12 Es decir, como un proceso socio-técnico, dinámico, auto-organizado, interactivo, de condicionamiento recíprocos, de mutua determinación, tensión, negociación y retroalimentación entre elementos heterogéneos: artefactos, actores, conocimientos, regulaciones, usuarios, políticas, formas de funcionamiento (Oudshoorn y Pinch, 2005, Vercelli y Thomas, 2007; Thomas 2008; Vercelli, 2009) 
necesarias para la gestión de estos derechos ${ }^{13}$. Las experiencias de gobierno abierto no dejan de ser gobiernos electrónicos mediados por la relación entre las regulaciones y las tecnologías digitales, Internet y redes móviles. En este sentido, las regulaciones y las formas de gestión de derechos ciudadanos se analizan como instancias tecnológicas, como artefactos tecnológicos, como mediaciones socio-técnicas. A su vez, correspondientemente, las tecnologías digitales, el software, los códigos digitales, las aplicaciones web o las plataformas de gobierno abierto se analizan como instancias regulativas.

\section{La participación política ciudadana y su institucionalización}

La participación política ciudadana es uno de los cuatro elementos descritos sobre los que se asientan y construyen los gobiernos abiertos. Esta participación política ciudadana bien puede considerarse su piedra basal y fuente de la soberanía popular. Justamente, es la participación política la que debería favorecer, permitir y construir los diferentes gobiernos (sean éstos más o menos abiertos). En la era digital, si hay gobiernos abiertos, entonces éstos tienen que nacer, articularse, sustentarse y co-construirse a través de las diversas formas de participación política de sus ciudadanías. Sin embargo, a diferencia de la transparencia y la gestión bienes intelectuales, la participacion política ciudadana ha sido uno de los temas con menos cambios evidentes dentro de los casos más resonantes de gobiernos abiertos a nivel mundial. No deja de sorprender esta situación.

El sentido común indica que a medida que crece la inclusión digital, a medida que aumenta la cantidad de ciudadanos que pueden expresarse y ejercer sus derechos a través de los medios electrónicos, la participación política ciudadana también debería hacerlo y podría verse enriquecida y comenzar a atravesar un proceso de renacimiento ${ }^{14}$. Sin embargo, no existen grandes cambios en relación a la participación en los gobiernos

\footnotetext{
${ }^{13}$ Las relaciones entre regulaciones y tecnologías en las formas de gestión de los entornos digitales abrió nuevos interrogantes tanto a nivel global como a nivel local / regional. Así, si las formas políticas para regular los sistemas de gestión de derechos ya no son sólo leyes, entonces, ¿qué actores / grupos sociales están capacitados para participar en su gestión? ¿Quiénes pueden desarrollar estas nuevas instancias de gestión basadas en el diseño de la tecnología? ¿Qué ocurre con las asimetrías existentes entre los países más desarrollados (y sus empresas transnacionales) y los países menos desarrollados? La articulación entre regulaciones de derechos intelectuales y las tecnologías digitales necesarias para expresar y ejecutar esos derechos comenzó a analizarse como un proceso político (socio-técnico) donde se codifican valores, posiciones e intereses de los diferentes grupos sociales involucrados (Vercelli y Thomas, 2008; Vercelli, 2009).

${ }^{14}$ ¿La participación política ciudadana es sinónimo de voto electrónico? El voto popular es, sin dudas, una de las formas de participación política: es, hasta el momento, la forma de participación política por antonomasia. Sin embargo, el voto y las urnas elecrónicas son un tema bien diferente y, en muchos puntos, forman parte de soluciones indeseables y riesgosas para el futuro de las democracias a nivel global.
} 
abiertos. Este es, justamente, uno de los puntos que debe ser profundizado a través del analisis de casos. Es posible que a nivel internacional todavía no esté muy claro qué es y cómo se da la participación política ciudadana. El análisis socio-técnico permitirá conocer si la participación política en los gobiernos abiertos es sólo una quimera.

Hoy existe la posiblidad real de que cualquier ciudadano (incluido tecnológicamente) pueda armar en minutos una página web, un blog o suscribirse a una red social que lo habilite a expresarse y le permita recibir comentarios, citas o referencias, favorece la construcción de diálogos distribuidos que cambian y complementan la relación política que la ciudadanía mantenía con los gobiernos y los grandes medios de comunicación (prensa, radios y televisión). En este sentido, las tecnologías digitales e Internet han permitido que la ciudadanía pueda expresarse y participar en el diálogo político utilizando los más diversos canales digitales, plataformas y redes sociales: los correos electrónicos, los foros de discusión, las cadenas de diapositivas, los salones de chat, la mensajería instantanea, los mensajes de texto, las páginas web y los blogs significaron y significan un cambio sustancial en este tipo de participación a nivel global. Algo similar ocurre con las diferentes formas de información, expresión y diálogo político que se producen a través de las plataformas comerciales y redes sociales como Facebook, Twitter, Youtube, Google Plus o Ning.

Sin embargo, rápidamente surgen profundos interrogantes. ¿Cualquier forma de expresión puede enterderse como una participación política ciudadana? ¿Todas las formas de participación mediadas por las tecnologías digitales son necesariamente buenas y deseables para las democracias? Entre otras preguntas, ¿expresar ideas, preferencias o discutir algo en una lista de distribución, foro o Twitter ya implica una participación política ciudadana? ¿La construcción de un foro temático en Facebook puede compararse con la elaboración de un presupuesto participativo y colaborativo? En igual sentido, ¿la participación política ciudadana sólo puede ser crítica y reactiva o también puede ser constructiva y alcanzar resultados institucionales tangibles? ¿Expresarse y opinar es igual a presentar formalmente un proyecto para reformar leyes, decretos 0 resoluciones?

Las nuevas formas de participación ciudadana pueden ir mucho más allá del ejercicio pleno de la libertad de expresión y del ejercicio constitucional de peticionar a las autoridades. ¿Es posible pensar en soluciones de gobierno abierto que permitan y favorezcan a los ciudadanos la gestión de otros derechos constitucionales? ¿Cuáles serían las tecnologías digitales, redes o plataformas que favorecerían estas nuevas formas de participación política ciudadana? Y, entonces, ¿existe casos de gobiernos abiertos con este nivel de gestión derechos ciudadanos? ¿Algún caso en la región sur del mundo?

A su vez, en relación a los canales de participación, ¿cualquier plataforma comercial puede servir a los fines de la participación ciudadana? ¿Es igual utilizar redes sociales comerciales masivas que canales 0 plataformas de las instituciones y partidos políticos de un país? Por ejemplo, 
¿cuántos canales y redes sociales institucionales se pueden enumerar en la UNASUR? ¿Cuántos de éstos ya están disponibles en Ministerios, Congresos, Direcciones Nacionales, Sindicatos, Municipios o Universidades? Ahora, ¿es conveniente institucionalizar estas nuevas formas de participación ciudadana? ¿Esta institucionalización favorecería mejores democracias o las limitaría? ¿Cuán institucional puede o debe ser esta participación ciudadana? ¿Un poco, mucho, completa? ¿Si la participación política ciudadana se institucionaliza se hace fuerte, se enriquece, pierde diversidad, se empobrece? Esta participación, ¿debe darse con nombre y apellido o también puede ser anónima?

\section{La gestión estratégica de los bienes intelectuales}

Otro de los elementos característicos de los gobiernos abiertos es la gestión de bienes intelectuales. A diferencia de lo que ocurre con la participación política ciudadana, la presencia de la gestión de datos, informaciones públicas y obras intelectuales es notoria y está presente en los principales casos referenciales de gobierno abierto a nivel mundial. Por ejemplo, Barack Obama comprendió rápidamente que la información del Gobierno Federal de los EE.UU. era parte de un "activo" o "patrimonio nacional" muy importante ["national asset"] (Obama, 2009). En poco más de un año de su administración los datos, informaciones públicas y otras obras intelectuales del Estado Federal pasaron a ser públicas y accesibles: en la actualidad pueden ser encontradas y utilizadas por los ciudadanos a través de portales web y otros servicios móviles.

Desde el primer día de gestión de Barack Obama el sitio web de la Casa Blanca comenzó a utilizar, para obras intelectuales de terceros ${ }^{15}$, una licencia abierta ${ }^{16}$ de 'Creative Commons' ${ }^{17}$ (White House, 2009). Específicamente, se utiliza la licencia de Atribución 3.0 de EE.UU. ${ }^{18}$. La nota

\footnotetext{
${ }^{15}$ En este caso obras intelectuales de terceros significa obras creadas u obras bajo titularidad de personas físicas o jurídicas diferentes del Estado de EE.UU. Por ejemplo, ciudadanos que comentan una entrada en un blog, que envían imágenes, que publican un artículo, etc. De acuerdo a la legislación de EE.UU., todas aquellas obras que aparecen en el sitio web de la Casa Blanca no están protegidas por Copyright (White House, 2009). Es decir, son obras intelectuales de carácter público y se ubican directamente en el dominio público.

${ }^{16}$ Una licencia es un instrumento legal [acuerdo / contrato] que expresa derechos de autor y que indica qué se puede y qué no se puede hacer con una obra intelectual. El licenciamiento es potestad del autor / autores de una obra o de quienes tengan su titularidad derivada. Se llaman abiertas a las licencias que crean obras intelectuales comunes del tipo abierto / libre (Vercelli, 2009).

17 'Creative Commons Corporation' es una organización no gubernamental, sin fines de lucro, fundada en 2001 en Estados Unidos de Norteamérica, que diseñó un sistema de licencias abiertas de 'liberación / reserva' selectiva de derechos de autor. Su sistema de licenciamiento abierto es voluntario. Existen otras licencias y sistemas de licencias abiertas / libres. Entre muchas otras, la Licencia de Documentación Libre (FDL-GNU), Arte Libre, Coloriuris o la licencia de Aire Incondicional. Sitio web de Creative Commons en http://www.creativecommons.org/
} 
de derecho de autor de la Casa Blanca y el uso de esta licencia indican que cualquier persona puede copiar, distribuir, hacer obras derivadas o explotar comercialmente las obras publicadas por terceros en el sitio de la Casa Blanca con la sola condición de atribuir la autoría en la forma especificada por el autor / titular derivado. La administracion Obama fue la primera en utilizar estas licencias y, rápidamente, otras siguieron sus pasos.

Países como el Reino Unido, Canadá, Australia, Holanda, Israel, Francia, Corea del Sur, Japón, España, Brasil, Chile, Uruguay o Argentina también se hicieron eco de estas iniciativas. Una de las primeras iniciativas en arribar a las modalidades de gobierno abierto fue el portal IREKIA del País Vasco. En enero de 2010 el Gobierno Vasco, España, creó 'IREKIA', un portal de gobierno abierto a la participación de la ciudadanía (Irekia, 2010b). El principal objetivo de IREKIA es permiterle a los ciudadanos interactuar con el Gobierno Vasco (Irekia, 2010). Los usuarios de la plataforma pueden hacer comentarios, discutir y hacer aportes mediante formas de trabajo colaborativas. Desde sus inicios este portal utilizó software libre ${ }^{19}$ y para la gestión de sus contenidos en línea optaron por la licencia abierta Creative Commons de Atribución 3.0 de España ${ }^{20}$ (Irekia, 2010b).

Algo similar ocurrió con el licenciamiento del portal del Gobierno Federal de Australia y los intentos de abrir bajo estas modalidades todo el 'Sector de Información Pública' (Australian Government, 2010). En abril de 2010 el Gobierno Australiano optó por el uso de la licencia abierta Creative Commons de Atribución 2.5 de Australia ${ }^{21}$. También por el mes de abril de 2010 se conoció la noticia de que el Gobierno de Holanda optó por una solución abierta para licenciar sus contenidos. A diferencia de los anteriores, en el caso de los portales del Gobierno de Holanda [Rijksoverheid] se escogió el uso de una dedicación de las obras al dominio público. Puntualmente, comenzaron a utilizar la dedicación de Creative Commons llamada $\mathrm{CCO}^{22}$ (Rijksoverheid, 2010).

En América Latina las iniciativas de gobierno abierto no se hicieron esperar. El caso más notorio de gobierno abierto fue el del Estado de San Pablo, Brasil. A través del Decreto № 53.963 / 2009 el Estado de San Pablo procuró desarrollar nuevas normativas sobre la gestión de la innovación y el

\footnotetext{
${ }^{18}$ Licencia disponible en http://creativecommons.org/licenses/by/3.0/us/

${ }^{19}$ Richard Stallman (2004) define el software libre a través de cuatro libertades en cabeza de los usuarios: [0] la libertad de usar el programa con cualquier propósito (libertad 0), [1] la libertad de estudiar cómo funciona el programa y adaptarlo a las necesidades (libertad 1). En este caso el acceso al código fuente es una condición previa para el ejercicio de esta libertad, [2] la libertad de distribuir copias y de esta forma ayudar a tu vecino (libertad 2), [3] la libertad de mejorar el programa y hacer públicas estas mejoras en beneficio de toda la comunidad (libertad 3). En este caso el acceso al código fuente es un requisito previo para el ejercicio de esta libertad.

${ }^{20}$ Licencia disponible en http://creativecommons.org/licenses/by/3.0/es/

${ }^{21}$ Licencia disponible en http://creativecommons.org/licenses/by/2.5/au/

${ }^{22}$ Licencia CCO disponible en http://creativecommons.org/publicdomain/zero/1.0/
} 
conocimiento (Agune, et. al., 2010). El Estado de San Pablo inició dos portales: uno orientado al ciudadano y otro orientado a la gestión de información y datos públicos. Algo similar ocurrió en Uruguay con la Intendencia de Montevideo. A través de la resolución 640 del año 2010 la Intendencia habilitó nuevas formas abiertas en la gestión de la información y los datos públicos (Res. 640, 2010). Los Chilenos desarrollaron su portal de Gobierno Abierto en 2011. En Argentina el gobierno abierto también comenzó a discutirse en 2009 (Calderón y Lorenzo, 2010), pero el gobieno federal no desarrollo aún una iniciativa específica al respecto.

En el mes de mayo de 2009 Barack Obama lanzó un proyecto complementario sobre la regulación de datos e informaciones provenientes del Gobierno Federal de EE.UU. Al igual que ocurrió con las obras intelectuales, la apertura / libertad proveniente de la cultura de Internet se hizo presente en la gestión de los bienes intelectuales dentro del proyecto Data.gov $^{23}$. Su principal objeto fue mejorar el acceso y la disponibilidad de los datos / informaciones generados por el Gobierno Federal que no involucren datos personales o información de seguridad nacional. A su vez, buscó generar ideas innovadoras para la gestión de estos datos y dar mayor transparencia, efectividad y apertura a su gestión de gobierno (Data.gov, 2009a). Al estar compuesto por información pública del Gobierno Federal de EE.UU. los datos / informaciones no se consideran alcanzados por el copyright dentro de los EE.UU. y, técnicamente, se encuentran en el dominio público de EE.UU. (Data.gov, 2009b).

Imitando la iniciativa de EE.UU., la administración del entonces Primer Ministro del Reino Unido, Gordon Brown, lanzó en enero de 2010 una iniciativa bajo la denominación Data.gov.uk ${ }^{24}$. El proyecto también gestiona datos e informaciones (que no son personales ni involucran temas de Seguridad Nacional) de la gestión pública. A diferencia del proyecto de EE.UU., el proyecto del Reino Unido desarrolló una licencia específica, que, a su vez, es compatible con la licencia de Atribución 3.0 'Unported' ${ }^{25}$ de 'Creative Commons Corporation'. La compatibilidad de las licencias facilita la interoperabilidad de los datos / informaciones y su reutilización (Data.gov.uk, 2010; Park, 2010). Sin embargo, según se anunció en el lanzamiento (Ellis, 2010), el gobierno del Reino Unido está preparando una nueva licencia, algo más específica, para gestionar obras intelectuales pero también los derechos sui generis sobre las bases de datos (Ellis, 2010).

Además de las iniciativas de EE.UU. y del Reino Unido, también los gobiernos Australia, Holanda, Nueva Zelanda y del País Vasco están desarrollando sus iniciativas sobre apertura de datos. En el caso del gobierno de Australia se ha desarrollado el sitio http://data.australia.gov.au/ para el tratamiento de todo tipo de datos referidos a la administración pública. Este sitio comparte la nota de derecho de autor del Portal del

\footnotetext{
${ }^{23}$ Sitio disponible en http://data.gov

${ }^{24}$ Sitio disponible en http://data.gov.uk

${ }^{25}$ Licencia disponible en http://creativecommons.org/licenses/by/3.0/
} 
Gobierno Federal, es decir, utiliza para gestionar los datos una licencia Creative Commons de Atribución 2.5 de Australia ${ }^{26}$. Lo mismo ocurre con la plataforma de datos que gestiona el Gobierno Vasco. A través de IREKIA se ha desarrollado el sitio de 'Open Data Euskadi' ['Datos Abiertos Euskadi'] en http://opendata.euskadi.net/. En este caso también se utiliza la licencia abierta Creative Commons de Atribución 3.0 de España ${ }^{27}$ (Open Data Euskadi, 2010). Finalmente, el uso de la dedicación al dominio público que implica CCO en el caso del portal de Gobierno de Holanda, indica que también están proyectado la misma solución para gestionar datos de una forma abierta.

En América Latina existen tres iniciativas claras sobre el tratamiento de informaciones públicas y datos abiertos. Como antes se mencionó, el Estado de San Pablo, Brasil, inició en 2009 un portal sobre la gestión abierta de datos: http://www.governoaberto.sp.gov.br/. Esta iniciativa se creo a través del Decreto № 55.559, del 12 de marzo de 2010, que ofrece a los ciudadanos -a través de Internet y otras redes electrónicas- la actualización y la disponiblidad (de forma abierta y gratuita) de las bases de datos públicas del Estado de San Pablo. El otro caso sobre datos abierto que puede mencionarse es el de República Oriental del Uruguay a través del portal http://datos.gub.uy/. Allí se destacan las iniciativas de Compras en el Estado y el antes mencionado caso de datos abiertos de la Intendencia de Montevideo. En el portal de la Intendencia de Montevideo se expresa que están evaluando la apertura de datos y que, en principio, los datos no estarán sujetos a ningún tipo de regulación de derechos, patentes o registros de marca (Intendencia de Montevideo, 2011). En 2011 se sumó el caso chileno a la gestión de datos abiertos: http://datos.gob.cl/

Los casos descritos permiten observar diferentes soluciones sobre la gestión estratégica de los bienes intelectuales. Permiten analizar como los incipientes gobiernos abiertos a nivel mundial también están influenciados por las formas abiertas / libres de gestionar los bienes intelectuales en la era digital. En otras palabras, estos casos permiten analizar como las formas abiertas y libres que provienen de la cultura de Internet forman parte de la construcción de las nuevas formas de gobierno. Las iniciativas del Gobierno Federal de EE.UU., tanto de la Casa Blanca como de Data.gov, muestran la relevancia que tiene la institución del dominio público en EE.UU. y como el uso de licencias abiertas complementa el carácter de los bienes intelectuales ${ }^{28}$. Como se describió, casos muy similares pueden observase en las iniciativas del Reino Unido, España (Irekia), Holanda, Australia, Brasil (San Pablo) e, incipientemente, en Uruguay (Montevideo) y Chile.

\footnotetext{
${ }^{26}$ Licencia disponible en http://creativecommons.org/licenses/by/2.5/au/

${ }^{27}$ Licencia disponible en http://creativecommons.org/licenses/by/3.0/es/

${ }^{28}$ El carácter que pueden adquirir los bienes permite definir cómo las personas / grupos sociales son dueños, titulares, poseedores, tenedores, autores, usuarios, etc. Esto permite saber como se disponen y se gestionan los bienes intelectuales.
} 
Ahora bien, en la República Argentina, ¿pueden desarrollarse experiencias similares sobre gestión de bienes intelectuales orientados a la construcción de gobiernos abiertos? ¿Las leyes nacionales, regulaciones y tecnologías de gestión permiten avanzar hacia un gobierno abierto en materia de gestión de datos, informaciones públicas y obras intelectuales? La República Argentina posee diversas normas relacionadas con la gestión de los bienes intelectuales que afectarían de forma directa e inmediata la construcción de gobiernos abiertos. A continuación se analizan brevemente las leyes argentinas relativas a la gestión de datos, informaciones pública y obras intelectuales.

En relación a la gestión de datos, Argentina cuenta con la Ley Nacional 25.326, también conocida como Ley de Habeas Data (Arg.PDP, 2000). Esta Ley regula el sistema de protección de datos personales (de personas físicas o jurídicas) en archivos, registros, bases de datos, bancos de datos, u otros medios técnicos de tratamiento de datos (sean éstos públicos o privados). Sin embargo, esta norma no alcanza a cubrir el desafío que representa el gobierno abierto en el tratamiento de datos / informaciones. Esta Ley está abocada a proteger datos personales sensibles de las personas físicas o de existencia ideal, pero no está preparada para abrir, compartir y gestionar datos, incluso los que tienen relevancia comercial, para los ciudadanos, las empresas, las organizaciones sociales u otros Estados.

En relación a la información pública, la República Argentina dispone del Decreto 1172 / 03 de 'Mejora de la Calidad de la Democracia y de sus Instituciones' que garantiza y regula el derecho ciudadano a requerir, consultar y recibir información pública (Arg.AIP, 2003) ${ }^{29}$. Cercano a los principios de los gobiernos abiertos, el Decreto regula la publicidad de los actos de gobierno, la transparencia en la gestión y el derecho al libre acceso a la información pública. La principal falencia de esta normativa es que (más allá de ser un Decreto) sólo regula la esfera de funcionamiento y comunicación del ciudadano con el Poder Ejecutivo Nacional. Sería conveniente que una Ley Nacional avance sobre la gestión de la información pública en todo el Estado Nacional y se incluyan otro poderes: Legislativo, Judicial, Auditoría General de la Nación, Defensoría del Pueblo, Ministerio Público Nacional. Este sería un gran paso hacia la construcción de gobiernos más abiertos.

Finalmente, sobre las obras intelectuales, la República Argentina posee un sistema nacional de derecho de autor y derecho de copia con base en la Ley 11.723 / 33 y reformas (Arg.LPI, 1933) ${ }^{30}$. A partir de este

\footnotetext{
${ }^{29}$ Puede consultarse el sitio web de la 'Secretaría de la Gestión Pública' sobre 'Mejor Democracia'. Disponible en http://www.mejordemocracia.gov.ar/.

${ }^{30}$ El derecho de autor y derecho de copia tutela el hecho de la creación intelectual por parte de los seres humanos. Los autores son las personas físicas que crean obras intelectuales y, por tanto, son sus titulares originarios. Los tratados, leyes y las normas sociales sobre derechos de autor y derecho de copia otorgan a los autores un conjunto de atribuciones y facultades para el ejercicio de sus derechos. Por un lado, le reconocen derechos personales / morales. Por el
} 
sistema en la Argentina se pueden definir, con relativa claridad, el carácter privativo $^{31}$ o común ${ }^{32}$ (abierto ${ }^{33}$ / libre ${ }^{34}$ ) que podrían alcanzar las obras intelectuales. Con algo más de dificultad, también se puede definir el carácter público de las mismas. En este sentido, una obra intelectual posee un carácter 'público' cuando ha sido creada por personas físicas en cumplimiento de funciones de gobierno (para las cuales han sido elegidas por el voto de la ciudadanía) o por personas físicas bajo relación de dependencia con el Estado (cuando éstas están en cumplimiento de sus funciones). El Estado, como persona jurídica, es siempre un titular derivado de derechos patrimoniales sobre las obras intelectuales ${ }^{35}$.

A pesar de las definiciones, sin embargo, en muchas ocasiones el carácter público de los datos, informaciones y obras intelectuales puede pasar a ser confuso, ambiguo y contradictorio. Podrían citarse varios ejemplos, aunque sólo se analizan dos. En primer lugar, se puede citar el artículo 27 de la Ley 11.723 que establece que “... Los discursos parlamentarios no podrán ser publicados con fines de lucro, sin la autorización del autor ..." (Arg.LPI, 1933). Este artículo desdibuja la idea de "lo público" en la función gubernamental por excelencia de los parlamentarios (Senadores y Diputados). Es decir, los ciudadanos no

otro, le reconocen derechos exclusivos para explotar económicamente su producción intelectual.

${ }^{31}$ Una obra intelectual tiene carácter 'privativo' cuando su autor o titular derivado mantiene la reserva de todos sus derechos de autor y no permite a los usuarios / comunidad más usos que los reconocidos como excepciones y limitaciones a sus derechos.

\footnotetext{
${ }^{32}$ Una obra intelectual tiene un carácter 'común' cuando ofrece a los usuarios de forma directa, inmediata y sin necesidad de solicitar permiso, la posibilidad de acceder, usar, copiar, distribuir y disponer de ella de acuerdo a mayores o menores limitaciones. Todas las obras intelectuales comunes permiten las capacidades antes descritas en manos de los usuarios y las comunidades.

${ }^{33}$ Las 'obras intelectuales comunes abiertas' son aquellas que, al momento de ser compartidas con las comunidades, sus autores o titulares derivados han decidido limitarlas en su explotación comercial o en su capacidad de generar obras derivadas (Lessig, 2007).

${ }^{34}$ Las 'obras intelectuales comunes libres' son aquellas que, al momento de ser compartidas con las comunidades, sus autores o titulares derivados han decidido permitir que los usuarios puedan usar la obra intelectual con cualquier propósito o finalidad, que puedan estudiarla y adaptarla a sus necesidades, que puedan copiar las obras y distribuirlas y que puedan derivar la obra y publicar estas obras derivadas (Stallman, 2004). Las obras intelectuales con carácter común libre tienen también una condición que, justamente, las transforma en libres: los autores o titulares derivados establecen como condición que las obras derivadas de la obra original continúen también bajo la misma licencia (que continúen siendo de carácter común libre). Este es el caso de las obras liberadas a través de licencias libres o copyleft.
}

35 En el derecho de autor argentino no hay disposiciones genéricas que presuman la transmisión de la titularidad (derivada) de las obras intelectuales por causa de la relación laboral. Sin embargo, queda claro que las obras que se produzcan en cumplimiento y desempeño de las funciones del trabajo para el cual el creador ha sido contratado establece una presunción de titularidad derivada sin afectar los derechos personales. En este caso los derechos sobre las obras intelectuales se transmiten al Estado a través de una relación de trabajo en relación de dependencia. 
pueden hacer un uso comercial directo e inmediato de las obras intelectuales (discursos parlamentarios) que son producidas por los legisladores en su función típica de gobierno. Este artículo desalienta el sistema democrático, representativo y republicano de gobierno. Por supuesto, tampoco construye gobiernos abiertos.

En segundo lugar, la ambigüedad sobre el carácter público también surge de la institución del dominio público oneroso (o pagante) al que está sujeto el derecho de autor y derecho de copia en la República Argentina (Arg.FNA, 1958). Vencido el plazo de protección del dominio privativo (70 años luego de la muerte del autor o 50 luego de la publicación en las obras anónimas pertenecientes a instituciones, corporaciones 0 personas jurídicas) las obras intelectuales caen indefectiblemente en el dominio público (Arg.LPI, 1933). Sin embargo, el componente oneroso / pagante del dominio público desvirtúa la libre disponibilidad de las obras intelectuales y establece el pago de un gravamen obligatorio (para el uso de las obras) a favor del Fondo Nacional de las Artes (Arg.FNA, 1958). El componente oneroso / pagante agravia el carácter público de los datos, informaciones y obras intelectuales (Librecultura, 2006). Más aún, obliga al Estado Argentino a producir sólo obras intelectuales dentro del dominio privativo y a indisponibilizar aquello que es de los ciudadanos.

\section{Hacia la construcción de mejores democracias en la era digital}

Los gobiernos abiertos no dejan de ser gobiernos electrónicos. Lejos de una plácida y descomprometida neutralidad tecnológica, las tecnologías digitales (y las tecnologías en general) traducen, codifican y solidifican estados de relaciones e intereses. Tienen una historia, un presente y un futuro. Así, las tecnologías con las que se diseñan y construyen los gobiernos y ciudadanías abiertas alrededor del mundo también codifican tensiones, negociaciones, luchas e intereses de los diferentes grupos sociales. La apertura descrita en las nuevas formas de gobierno es, sin dudas, tributaria de la cultura abierta de Internet, de su arquitectura política, de las luchas por el software abierto / libre y de las luchas por la liberación de la cultura en todas sus formas. Este no es un tema menor, cierto nivel explicativo sobre el concepto de gobierno y ciudadanías abiertas depende de estas tradiciones.

Las arquitectura distribuida, abierta y de producción colaborativa entre pares que definió y todavía define la red de redes marca un punto clave para el análisis de los gobiernos abiertos. Los gobiernos abiertos ¿nacen de un repollo o también son una construcción político-tecnológica? Los gobiernos abiertos no se producen por generación espontánea ni pueden transferirse desde otras realidades socio-históricamente situadas. Éstos son una construcción situada y cotidiana, son un emergente de producción colaborativa. Se construyen diariamente a partir del aporte de cada uno de sus ciudadanos. En este sentido, los gobiernos abiertos serán un emergente de producción colaborativa o no serán. 
Por ello, analizar el aporte ciudadano (individual o colectivo) será central al momento de estudiar la construcción de estas nuevas formas abiertas de gobierno / ciudadania. La construcción socio-técnica de los gobiernos abiertos debe encontrar su correlato en nuevas formas abiertas de las ciudadanías. Esta línea de investigación debe indagar y analizar si, correspondientemente, también se están co-construyendo nuevas formas de ejercer y gestionar derechos ciudadanos. ¿Se están desarrollando también ciudadanías abiertas en la era digital? La aparente inmutabilidad de la participación política ciudadana es una de las claves que debe ser analizada empiricamente. La participación política de la ciudadanía es sin dudas el punto más importante de los gobiernos abiertos. Estas nuevas formas de participación deben poder caracterizarse y analizarse dentro de marcos más institucionales.

Con mucha mayor visibilidad, la gestión de bienes intelectuales se ha transformado en una pieza estratégica para el desarrollo político, económico, institucional y social a nivel global. La construcción de los gobiernos y ciudadanías abiertas no puede obviar este cuarto elemento. Las iniciativas analizadas muestran que diferentes administraciones a nivel mundial, sobre todo en EE.UU., comienzan a dejar libremente accesible y disponible aquello que pertenece a los ciudadanos, a la comunidades, a las sociedades en su conjunto, aquello que, de una u otra forma, ha sido financiado con fondos públicos. La construcción de los gobiernos abiertos en la Argentina debe, necesariamente, avanzar sobre una nueva caracterización de "lo público". En este sentido, se deben revisar exhaustivamente las diferentes leyes y legislar con perspectiva de futuro. Legislar reivindicando el sentido público de la política. Es necesario reconsiderar el dominio público pagante / oneroso y avanzar sobre el desarrollo local de tecnología y licencias abiertas.

Las nuevas formas de producción y gestión de estos bienes intelectuales comienzan a afectar de forma directa las iniciativas de gobiernos abiertos. Por su calidad, los bienes intelectuales, estas formas de valor, son abundantes y pueden compartirse libremente entre diferentes grupos sociales sin generar relaciones de escasez. De allí que la gestión de los derechos intelectuales sea, sin dudas, uno de los puntos estratégicos fundamentales al momento analizar los gobiernos y ciudadanías abiertas. El libre acceso y disponibilidad a estos bienes intelectuales es fundamental para el ejercicio directo de otros derechos ciudadanos. Una parte sustancial de la democracia comienza a pasar por el ejercicio y la gestión de estos derechos intelectuales. El análisis de estas formas de gestión permite conocer de forma directa y clara qué tipo democracias se están construyendo en la era digital.

Hasta el momento estos análisis han tenido una peligrosa desatención en las agendas político-científicas de los países en desarrollo. Las nuevas regulaciones vinculadas al diseño y producción de tecnologías impactan directamente sobre la autonomía, la soberanía y la defensa de los intereses nacionales / regionales. Van construyendo aspectos significativos del desarrollo y democratización de procesos decisorios a nivel global / 
regional. La construcción de gobiernos y ciudadanías abiertas se ha transformado en un tema estratégico para Argentina y toda América Latina. Las relaciones que se producen entre el ejercicio de los derechos ciudadanos y las tecnologías digitales diseñadas para su gestión pueden definir (y así lo hacen) aspectos importantes del futuro político, cultural, social y económico de las sociedades. Es necesario que Argentina y otros países de América Latina desarrollen sus propias soluciones de gobierno abierto y que éstas respondan a sus identidades, formas productivas, industrias, intereses nacionales y, en suma, que sean parte de una estrategia basada en la soberanía nacional / regional y en la integración regional.

La construcción de los gobiernos y ciudadanías abiertas en la República Argentina y la región sur del mundo debe estar guiada por la apertura, la libertad y la disponibilidad del software libre. Los gobiernos y ciudadanías abiertas deben construirse sobre las bases de la soberanía tecnológica. Es importante desarrollar soluciones tecnológicas (software, hardware, regulaciones) que sean libres, abiertas, públicas y comunitarias. Es importante alentar el diseño colaborativo de estrategias tecnológicoregulativas locales y regionales que se articulen, converjan y formen parte del desarrollo de soluciones más equitativas para gestionar los derechos ciudadanos. Para ello, esta investigación debe continuar a través de la producción de nuevo conocimiento de base empírica que permita entender cómo funcionan o dejan de funcionar los gobiernos abiertos y que permita generar diferentes recomendaciones: políticas nacionales sobre gobiernos abiertos, desarrollo de modelos de gestión de derechos ciudadanos, propuestas legislativas, reformas de leyes o formulación de estrategias, alianzas y posicionamientos de Argentina y América Latina en organismos internacionales.

\section{Referencias}

AGUNE, R., GREGORIO FILHO, A., BOLLIGER, S. (2010, mayo). Governo Aberto SP: Disponibilizacao de bases de dados e informacoes em formato aberto. Presentado en el III Congreso de Consad de Gestao Pública en mayo de 2010. Disponible en http://www.repositorio.seap.pr.gov.br/arquivos/ File/Material 2520CONSAD/paineis III congresso consad/painel 13/gover no aberto $\mathrm{sp}$ disponibilizacao de bases de dados e informacoes em $f$ ormato aberto.pdf.

Argentina. Ley de Propiedad Intelectual. Ley 11.723 (1933). Disponible texto actualizado en http://www.infoleg. gov.ar/infoleglnternet/anexos/4000044999/42755/texact.htm.

Argentina. Creación del Fondo Nacional de las Artes. Decreto-Ley 1.224 (1958). Disponible en http://www.a-d-a.com.ar/descargas/decretoley122458.pdf. 
Argentina. Protección de Datos Personales. Ley 25.326. (2000). Disponible texto actualizado en http://www.info leg.gov.ar/infolegInternet/anexos/6000064999/64790/texact.htm.

Argentina. Acceso a la Información Pública. Decreto 1172 (2003). Disponible en http://www.infoleg.gov.ar/ infoleglnternet/anexos/9000094999/90763/norma.htm.

Australian Government. Copyright. Disponible en http://australia.gov.au/about/copyright. 2010.

BARLOW, J., P. The Economy of Ideas: A framework for patents and copyrights in the Digital Age (Everything you know about intellectual property is wrong). Disponible en http://www.wired.com/wired/archive/2.03/economy.ideas.html. 1994.

BARLOW, J., P. A Declaration of the Independence of Cyberspace. Disponible en http://www.eff.org/ barlow/Declaration-Final.html. 1996.

BENKLER, Y. The wealth of the networks: How Social Production Transforms Markets and Freedom. Estados Unidos de Norteamérica: Yale University Press. 2006.

BERNERS Lee, T. Tejiendo la red: el inventor del World Wide Web nos descubre su origen. Madrid: Siglo XXI España. 2000.

BIJKER, W. Of Bicycles, Bakelites, and Bulbs: Toward a Theory of Sociotechnical Change. Cambridge, MA: MIT Press. 1995.

CALDERÓN, C y LORENZO, S. 'Open Government - Gobierno Abierto', Algón Editores, Alcalá la Real (Jaen). 2010.

CALLON, M. Redes tecno-económicas e irreversibilidad. Em Revista Redes, 17, pp. 83-126. Bernal, Buenos Aires: Universidad Nacional de Quilmes. 1998.

CASTELLS, M. Comunicación y poder. Madrid: Alianza. 2009.

CASTELLS, M. La Galaxia Internet. Madrid: Areté. 2011.

Data.gov. About. Sitio web de Data.gov. Disponible en http://www.data.gov/about. 2009a

Data.gov Data Policy. Sitio web de Data.gov. Disponible en http://www.data.gov/datapolicy. 2009b

Data.gov.uk. Terms and Conditions. Sitio web de Data.gov.uk. Disponible en http://data.gov.uk/terms-conditions. 2010. 
ELLIS, J. Licensing and data.gov.uk launch. Sitio web Perspectives. Disponible en http://perspectives.opsi.gov.uk/2010/01/licensing-anddatagovuk-aunch.html. 2010.

FEENBERG, A. Questioning Technology. New York: Editorial Routledge. 1999.

FEENBERG, A. Transforming Technology: A Critical Theory Revisited. New York: Oxford.

Kapor, M. (1990). The Software Design Manifesto. Disponible en http://hci.stanford.edu/bds/1-kapor.html. 2002.

Intendencia de Montevideo. Principios para el manejo de datos abiertos en el gobierno. Disponible en http://www.montevideo.gub.uy/institucional/datos-abiertos/principios-para-elmanejo-de-datos-abiertos-en-el-gobierno. 2011.

IREKIA. Irekia, Gobierno Abierto. Sitio Web de Irekia, Gobierno Vasco. Disponible en http://www.irekia.euskadi.net/es/pages/798-acerca. 2010a

IREKIA. Irekia, Gobierno Abierto. Sitio Web de Irekia, Gobierno Vasco. Disponible en http://www.irekia.euskadi.net/. 2010b

LATOUR, B. Jamais Fomos Modernos. Rio de Janeiro: Editora 34. 1994.

LESSING, L. The future of the ideas: the fate of the commons in a connected world. Nueva York: Random House. 2001.

LESSING, L. Free Culture: How Big Media Uses Technology and the Law to Lock Down Culture and Control Creativity. Nueva York: Penguin Press. 2004.

LESSING, L. Code: Version 2.0. Nueva York: Basic Books. 2006.

LESSING, L. Retiring standalone DevNations and one Sampling license. Disponible en http://creativecommons.org/weblog/entry/7520. 2007.

LESSING, L. Remix: Making Art and Commerce Thrive in the Hybrid Economy. Nueva York: Penguin Press. 2008.

LIBRECULTURA. Manifiesto por la Librecultura: parte del movimiento por la liberación de la cultura en todas sus formas. Disponible en http://www.librecultura.org/manifiesto.html. Versión 1.1. del 17 de noviembre de 2008. 2006.

MITCHELL, W. City of Bits: Space, Place and Infobahn. Cambridge, MIT Press. 1995. 
NASER, A., CONCHA, G. Panorama de Gobierno Electrónico en la región: resultados e impactos, en Gastón Concha y Alejandra Naser Editores, Documentos de Proyectos de CEPAL, 'El desafío hacia el gobierno abierto en la hora de la igualdad', Naciones Unidas, Santiago de Chile. Disponible http://www.cepal.org/ddpe/publicaciones/xml/9/46119/W465.pdf. 2012.

en

OBAMA, B. Transparency and Open Government. Sitio Web de la Casa Blanca.

Disponible en

http://www.whitehouse.gov/the press office/Transparency and Open

Government/. 2009.

Open Data Euskadi. Qué es Open Data. Sitio Web del Gobierno Vasco. Disponible en http://opendata.euskadi.net/w79opendata/es/contenidos/informacion/que es opendata/es que es/que es opendata.html. 2010.

ORSZAG, P. Open Government Directive. Sitio web de la Casa Blanca. Disponible en http://www.whitehouse.gov/open/documents/opengovernment-directive. 2009.

OUDSHOORN, N., PINCH, T. How Users Matters: the co-construction of users and technology. Cambridge, MA: MIT Press. 2005.

PARK, J. UK moves towards opening government data. Sitio web de Creative Commons. Disponible en http://creativecommons.org/weblog/entry/20228. 2010.

RAMíREZ ALUJAS, A. Innovación en la Gestión Pública y Open Government (Gobierno Abierto): Una vieja nueva idea... . Revista Buen Gobierno, № 9, Jul-Dic 2010, p. 2-35. 2010.

Resolucion 640, de la Intendencia de Montevideo, Uruguay. Disponible en http://monolitos.montevideo.gub.uy/resoluci.nsf/de053405568724cf832575a e004f0467/7adaf8ec8d70033b832576d60041760f. 2010.

RIJKSOVERHEID. Copyright: Auteursrechtelijke informatie over de inhoud van deze website. Disponible en http://www.rijksoverheid.nl/copyright. 2010.

STALLMAN, R., M. Software libre para una sociedad libre. Traficantes de Sueños: Madrid. Disponible en versión digital en http://www.traficantes.net/index.php/trafis/content/download/ 18110/185232/file/softlibre\%20enriquecido.pdf. 2004. 
THOMAS, H. Dinâmicas de inovação na Argentina (1970-1995): Abertura comercial, crise sistémica e rearticulação. Tesis de Doctorado, Universidad Estadual de Campinas. 1999.

THOMAS, $\mathrm{H}$. Estructuras cerradas vs. procesos dinámicos: trayectorias y estilos de innovación y cambio tecnológico. En Hernán Thomas y Alfonso Buch (Eds), Actos, actores y artefactos: Sociología de la Tecnología. (pp. 217-262). Bernal: Universidad Nacional de Quilmes. 2008.

VERCELLI, A. La Conquista Silenciosa del Ciberespacio: Creative Commons y el diseño de entornos digitales como nuevo arte regulativo en Internet. Disponible en http://www.ariel vercelli.org/lcsdc.pdf. 2004.

VERCELLI, A. Aprender la Libertad: el diseño del entorno educativa y la producción colaborativa de los contenidos básicos comunes. Disponible en http://www.aprenderlalibertad.org/aprender lalibertad.pdf. 2006.

VERCELLI, A. Repensando los bienes intelectuales comunes: análisis socio-técnico sobre el proceso de co-construcción entre las regulaciones de derecho de autor y derecho de copia y las tecnologías digitales para su gestión. Tesis doctoral. Disponible en http://www.arielvercelli.org/rlbic.pdf. $\underline{2009}$.

VERCELLI, A., Thomas, $\mathrm{H}$. La co-construcción de tecnologías y regulaciones: análisis socio-técnico de un artefacto anti-copia de SonyBMG. Revista Espacios, 3, 5-30. 2007

VERCELLI, A., Thomas, H. Repensando los bienes comunes: análisis socio-técnico sobre la construcción y regulación de los bienes comunes. Versión 1.1. Disponible en http://www.bienescomunes.org/archivo/rlbc-1-1.pdf. 2008.

WHITE HOUSE. Copyright Policy. Disponible en http://www.whitehouse.gov/copyright. 2009 